\title{
Two particles in a double well: illustrating the connection between entanglement and the speed of quantum evolution
}

\author{
S Curilef ${ }^{1}$, C Zander $^{2}$ and A R Plastino ${ }^{2}$ \\ ${ }^{1}$ Departamento de Física, Universidad Católica del Norte, Antofagasta, Chile \\ ${ }^{2}$ Physics Department, University of Pretoria, Pretoria 0002, South Africa \\ E-mail: arplastino@maple.up.ac.za
}

Received 6 March 2006, in final form 29 June 2006

Published 31 July 2006

Online at stacks.iop.org/EJP/27/1193

\begin{abstract}
The connection between entanglement and the speed of quantum evolution (as measured by the time needed to reach an orthogonal state) is discussed in the case of two quantum particles moving in a one-dimensional double well. The aforementioned connection offers an interesting opportunity of discussing the basic features of quantum entanglement within an elementary context, using concepts and methods usually included in university courses of quantum mechanics.
\end{abstract}

\section{Introduction}

Quantum entanglement [1-12] is widely regarded as one of (if not the) most fundamental feature of the quantum description of Nature [13-15]. It has been the subject of intense research activity in recent years [16-27]. Quantum entanglement constitutes a physical resource playing a fundamental role in connection with important quantum information processes [15] such as quantum teleportation [16], superdense coding [17] and quantum computation [18]. The experimental implementation of these processes may have important practical applications, not only in the communication and computational technologies, but also in other areas, such as quantum metrology [19]. Besides its technological relevance, current research in quantum entanglement is shedding new light upon fundamental aspects of quantum physics, such as, for instance, the emergence of thermodynamic behaviour within composite quantum systems [21]. Another example is provided by an interesting relationship between entanglement and the time evolution of composite quantum systems that has recently been established [22-24]: quantum 


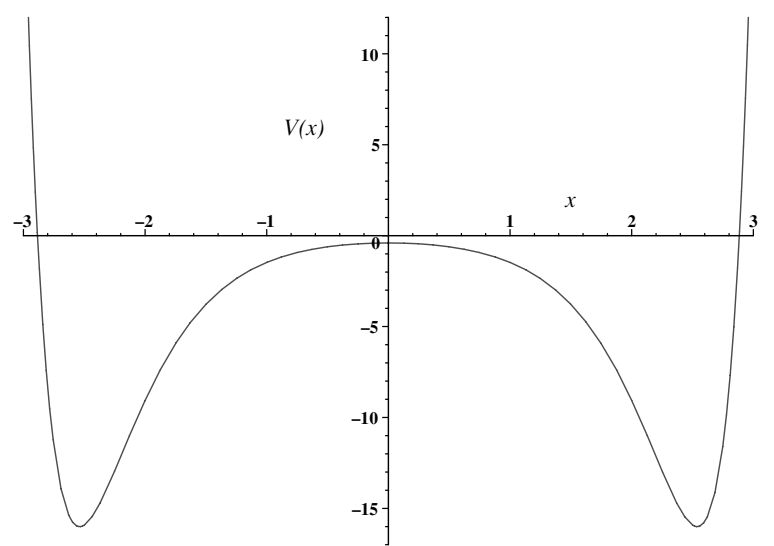

Figure 1. Double-well potential function $V(x)$. The potential $V(x)$, and the coordinate $x$ are depicted, respectively, in units of $\hbar^{2} \kappa^{2} / 2 m$ and $1 / \kappa$ (see the appendix for details).

entanglement enhances the 'speed' of evolution of certain quantum states, as measured by the time needed to reach an orthogonal state.

Due to its great importance, both from the fundamental and from the practical point of view, it is imperative to incorporate the concept of entanglement into the teaching of quantum mechanics [28, 29]. The aim of the present contribution is to illustrate some aspects of the relationship between quantum entanglement and the speed of quantum evolution, in connection with the problem of the tunnelling time in a double-well potential [30, 31]. The connection between entanglement and quantum evolution allows for a discussion of the basic aspects of quantum entanglement on the basis of ideas (such as the idea of tunnelling time) that are usually included in university courses of quantum mechanics. Consequently, the present discussion offers the important pedagogical advantage of not requiring a discussion of subtle topics in information theory, cryptography or computer science.

\section{Two particles in a double well: separable versus entangled states}

We are going to consider a system consisting of two distinguishable particles of mass $m$ (with coordinates $x_{1}$ and $x_{2}$ ) moving in a one-dimensional double-well potential $V(x)$ (see figure 1). The eigenstates associated with the potential $V(x)$ can be used, for instance, as an approximate description of the low-lying states of a homonuclear diatomic molecule [32]. Two such molecules constitute a possible physical realization of the bipartite composite quantum system that we are going to consider here. Details on the particular double-well potential used to generate the figures of this paper are given in the appendix. Let $\left|\psi_{0}\right\rangle$ and $\left|\psi_{1}\right\rangle$ denote the (one particle) ground and first excited states corresponding to the potential $V(x)$, with eigenenergies $E_{0}$ and $E_{1}$, respectively. The ground state $\left|\psi_{0}\right\rangle$ is described by an even wavefunction, while the state $\left|\psi_{1}\right\rangle$ is described by an odd wavefunction (see figure 2). The linear combinations

$$
\left|\psi_{R}\right\rangle=\frac{1}{\sqrt{2}}\left\{\left|\psi_{0}\right\rangle+\left|\psi_{1}\right\rangle\right\}
$$

and

$$
\left|\psi_{L}\right\rangle=\frac{1}{\sqrt{2}}\left\{\left|\psi_{0}\right\rangle-\left|\psi_{1}\right\rangle\right\}
$$




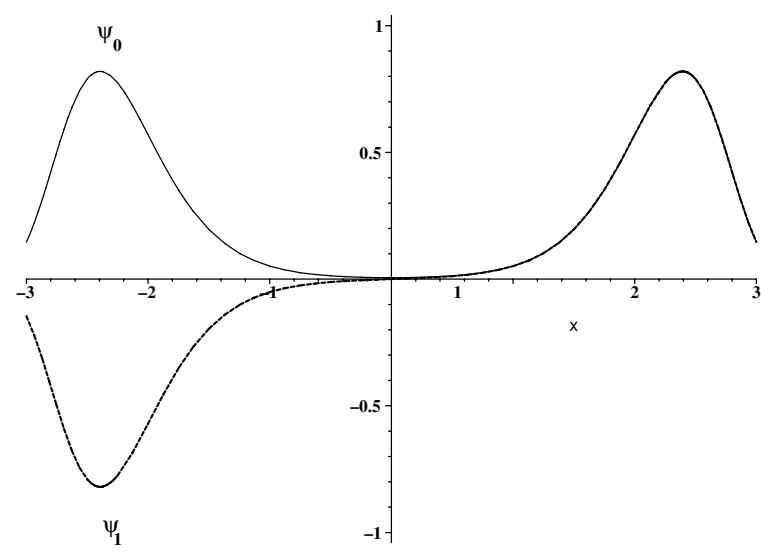

Figure 2. The ground state and first excited state wavefunctions of the double-well potential $V(x)$. The wavefunctions and the spatial coordinate $x$ are depicted, respectively, in units of $\sqrt{\kappa}$ and $1 / \kappa$ (see the appendix for details).

correspond to states of a particle localized, respectively, in the right-hand side well and in the left-hand side well of $V(x)$. If we consider only (single-particle) states that are linear combinations of $\left|\psi_{0}\right\rangle$ and $\left|\psi_{1}\right\rangle$, we have a system described by an effective two-dimensional Hilbert space or, in quantum information parlance, an effective 1-qubit system.

Now we are going to focus our attention on states of two distinguishable quantum particles living in the potential $V(x)$. We are going to work only with states that are linear combinations of the four states $\left|\psi_{0}\right\rangle\left|\psi_{0}\right\rangle,\left|\psi_{0}\right\rangle\left|\psi_{1}\right\rangle,\left|\psi_{1}\right\rangle\left|\psi_{0}\right\rangle$ and $\left|\psi_{1}\right\rangle\left|\psi_{1}\right\rangle$. In other words, we are going to work with an effective 2-qubit system. Pure states of this composite system can be classified into factorizable pure states and entangled pure states. On the one hand we have factorizable states

$$
|\phi\rangle=|\phi\rangle_{1} \otimes|\phi\rangle_{2},
$$

where $|\phi\rangle_{k}(k=1,2)$ are pure states of the $k$-particle. On the other hand we have entangled states, which are those that cannot be written in the form (3). In the case of factorizable states, each subsystem (in our case, each one of the two particles) is described by an individual pure state of its own (that is, the states $|\phi\rangle_{k}(k=1,2)$ in (3)). On the contrary, when we have an entangled state it is not possible to assign an individual pure state to each subsystem. In this case, the subsystems are in mixed states, which are described by density matrices. If our two-particle system is in a (pure) entangled state $|\psi\rangle$, each particle's state is described by a (marginal) density matrix obtained by taking the trace of the projector $|\psi\rangle\langle\psi|$ over the coordinate of the other particle. That is, the particles are described by density matrices $\rho_{k}$ given by

$$
\rho_{1}=\operatorname{Tr}_{2}(|\psi\rangle\langle\psi|), \quad \rho_{2}=\operatorname{Tr}_{1}(|\psi\rangle\langle\psi|) .
$$

More explicitly, the matrix elements of the density matrices $\rho_{k}$ are given by

$$
\begin{aligned}
& \left\langle x_{1}^{\prime}\left|\rho_{1}\right| x_{1}\right\rangle=\int\left\langle x_{1}^{\prime}, x_{2} \mid \psi\right\rangle\left\langle\psi \mid x_{1}, x_{2}\right\rangle \mathrm{d} x_{2}, \\
& \left\langle x_{2}^{\prime}\left|\rho_{2}\right| x_{2}\right\rangle=\int\left\langle x_{1}, x_{2}^{\prime} \mid \psi\right\rangle\left\langle\psi \mid x_{1}, x_{2}\right\rangle \mathrm{d} x_{1} .
\end{aligned}
$$


Not all entangled states are endowed with the same amount of entanglement. A quantitative measure of the amount of entanglement of a pure state $|\psi\rangle$ is given by the von Neumann entropy of either of the matrices $\rho_{k}$,

$$
\mathcal{E}(|\psi\rangle)=-\operatorname{Tr}\left(\rho_{1} \ln \rho_{1}\right)=-\operatorname{Tr}\left(\rho_{2} \ln \rho_{2}\right) .
$$

It is clear that according to the measure $\mathcal{E}(|\psi\rangle)$ factorizable pure states have zero entanglement.

A fundamental property of the measure of entanglement (6) is that it does not change under the action of local unitary transformations. That is, it does not change under the action of transformations of the form

$$
U=U_{1} \otimes U_{2},
$$

where $U_{1,2}$ represent unitary transformations acting on each particle individually.

In the problem we are considering, we have two non-interacting quantum particles moving in the same potential $V(x)$. The Hamiltonian of our system is of the form

$$
H=H_{1} \otimes I_{2}+I_{1} \otimes H_{2},
$$

where $I_{k}$ stands for the identity operator acting on the Hilbert space associated with the $k$-particle $(k=1,2)$ and

$$
H_{k}=-\frac{\hbar^{2}}{2 m} \frac{\partial^{2}}{\partial x_{k}^{2}}+V\left(x_{k}\right) \quad(k=1,2) .
$$

It is clear that the time evolution operator of this system is local (that is, it has the form (7)) with

$$
U_{k}=\exp \left[-\frac{\mathrm{i} t H_{k}}{\hbar}\right] \quad(k=1,2) .
$$

Consequently, the amount of entanglement of our two particles is not going to change in time. Note that the amount of entanglement exhibited by a given state of our two-particle system is an intrinsic property of that state. It does not depend on the form of the Hamiltonian. However, the Hamiltonian determines the evolution of the state and, consequently, also determines eventual changes in the amount of entanglement. In the case of the Hamiltonian (8)-(9) that we are going to consider, the amount of entanglement at any time is going to be the same as the amount of entanglement $\mathcal{E}\left(\left|\psi\left(t_{0}\right)\right\rangle\right)$ associated with the initial state $\left|\psi\left(t_{0}\right)\right\rangle$. Obviously, the initial entanglement $\mathcal{E}\left(\left|\psi\left(t_{0}\right)\right\rangle\right)$ is, in turn, determined by the particular way in which the initial state was prepared.

\section{Entanglement and the speed of quantum evolution}

\subsection{The speed of quantum evolution and its lower bound}

A natural measure for the 'speed' of quantum evolution is provided by the time interval $\tau$ that a given initial state $\left|\psi\left(t_{0}\right)\right\rangle$ takes to evolve into an orthogonal state [22-24, 33],

$$
\left\langle\psi\left(t_{0}\right) \mid \psi\left(t_{0}+\tau\right)\right\rangle=0
$$

In our present example involving two particles in a double-well potential, $\tau$ can be regarded as the tunnelling time of the two-particle system. Let $E$ denote the energy's expectation value,

$$
E=\langle H\rangle
$$

and $\Delta E$ denote the energy's standard deviation, defined as the square root of the variance, which measures the energy's uncertainty,

$$
\Delta E=\sqrt{\left\langle H^{2}\right\rangle-\langle H\rangle^{2}} .
$$




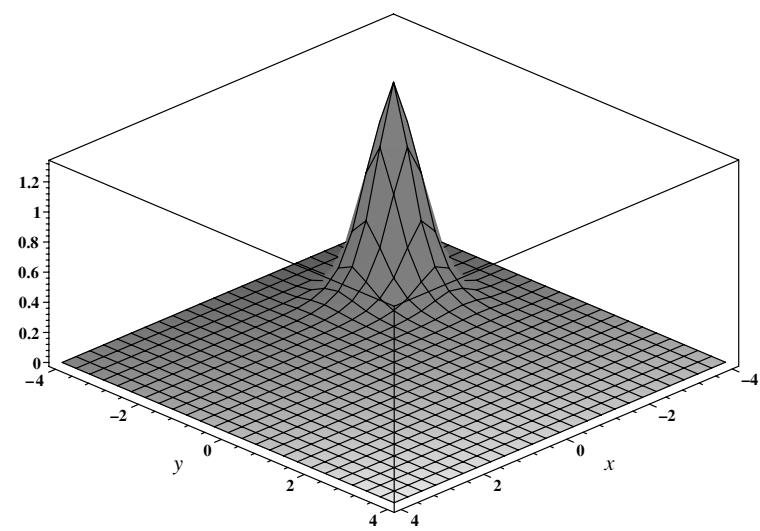

Figure 3. The wavefunction corresponding to the state $\left|\psi_{R R}\right\rangle$. The wavefunction and the spatial coordinates $x_{1,2}$ are depicted, respectively, in units of $\kappa$ and $1 / \kappa$ (see the appendix for details).

A lower limit for the evolution time $\tau$ to an orthogonal state is given by [33]

$$
\tau_{\min }=\frac{\pi \hbar}{2 \Delta E} \text {. }
$$

\subsection{Comparing a separable state and a maximally entangled state}

Let us first consider the factorizable, two-particle state

$$
\left|\psi_{R R}\right\rangle=\left|\psi_{R}\right\rangle \otimes\left|\psi_{R}\right\rangle
$$

corresponding to a situation where both particles are localized in the right-hand side well (see figure 3). The expansion of this state in the Hamiltonian's eigenbasis is

$$
\left|\psi_{R R}\right\rangle=\frac{1}{2}\left\{\left|\psi_{0}\right\rangle\left|\psi_{0}\right\rangle+\left|\psi_{0}\right\rangle\left|\psi_{1}\right\rangle+\left|\psi_{1}\right\rangle\left|\psi_{0}\right\rangle+\left|\psi_{1}\right\rangle\left|\psi_{1}\right\rangle\right\}
$$

the energy expectation value is

$$
E_{R R}=E_{0}+E_{1},
$$

and the energy uncertainty is

$$
\Delta E_{R R}=\frac{1}{\sqrt{2}}\left(E_{1}-E_{0}\right) .
$$

The concomitant lower bound for the time to reach an orthogonal state is then (see equation (14))

$$
\tau_{\min }\left(\left|\psi_{R R}\right\rangle\right)=\frac{\hbar \pi}{2 \Delta E_{R R}}=\frac{\hbar \pi}{\sqrt{2}\left(E_{1}-E_{0}\right)} .
$$

On the other hand, the time actually needed by state $\left|\psi_{R R}\right\rangle$ to evolve into the orthogonal state $\left|\psi_{L L}\right\rangle=\left|\psi_{L}\right\rangle \otimes\left|\psi_{L}\right\rangle$ (whose wavefunction is depicted in figure 4) is the same as the time needed by the one-particle state $\left|\psi_{R}\right\rangle$ to evolve to $\left|\psi_{L}\right\rangle$,

$$
\tau\left(\left|\psi_{R R}\right\rangle\right)=\tau\left(\left|\psi_{R}\right\rangle\right)=\frac{\hbar \pi}{\left(E_{1}-E_{0}\right)}
$$

Consequently,

$$
\tau_{\min }\left(\left|\psi_{R R}\right\rangle\right)=\frac{1}{\sqrt{2}} \tau\left(\left|\psi_{R R}\right\rangle\right)<\tau\left(\left|\psi_{R R}\right\rangle\right)
$$




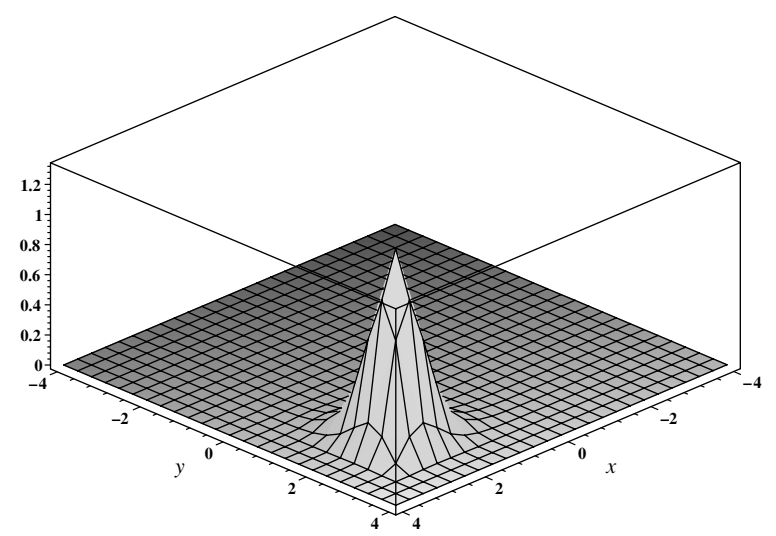

Figure 4. The wavefunction corresponding to the state $\left|\psi_{L L}\right\rangle$. The wavefunction and the spatial coordinates $x_{1,2}$ are depicted, respectively, in units of $\kappa$ and $1 / \kappa$ (see the appendix for details).

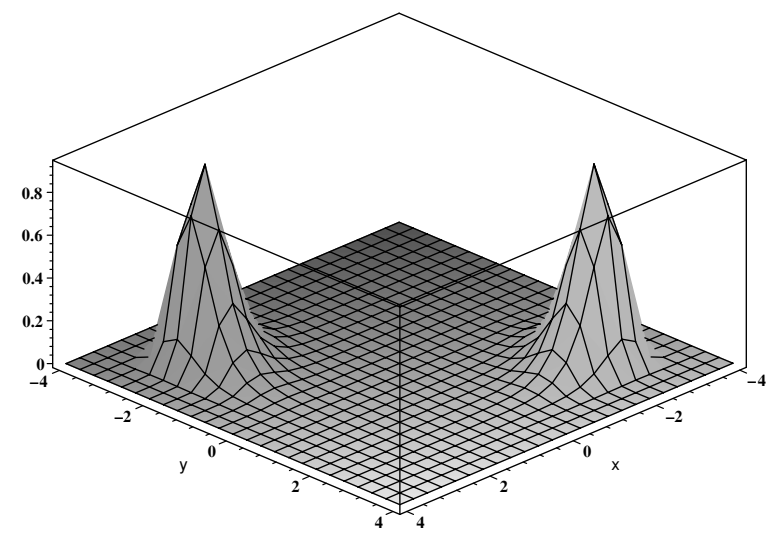

Figure 5. The wavefunction corresponding to the state $\left|\psi_{\text {ent }}\right\rangle$. The wavefunction and the spatial coordinates $x_{1,2}$ are depicted, respectively, in units of $\kappa$ and $1 / \kappa$ (see the appendix for details).

Therefore, we see that the state $\left|\psi_{R R}\right\rangle$ does not saturate the bound (14). Its evolution is not as fast as it is allowed by the bound (14). Let us now consider the entangled state

$$
\left|\psi_{\text {ent }}\right\rangle=\frac{1}{\sqrt{2}}\left(\left|\psi_{0}\right\rangle\left|\psi_{0}\right\rangle+\left|\psi_{1}\right\rangle\left|\psi_{1}\right\rangle\right)
$$

whose energy expectation value is

$$
E_{\text {ent }}=E_{0}+E_{1}
$$

and whose energy uncertainty is

$$
\Delta E_{\text {ent }}=E_{1}-E_{0} .
$$

The state $\left|\psi_{\text {ent }}\right\rangle$ is (within our effective 2-qubit system) maximally entangled. The amount of entanglement of this state is (see equation (6))

$$
\mathcal{E}\left(\left|\psi_{\text {ent }}\right\rangle\right)=\ln 2,
$$

which is the maximum amount of entanglement in a 2-qubit system.

The wavefunction of state $\left|\psi_{\text {ent }}\right\rangle$ is exhibited in figure 5. After a time interval $\tau\left(\left|\psi_{\text {ent }}\right\rangle\right)$, this state evolves into the orthogonal state $\left|\psi_{\text {ent }}\right\rangle^{\perp}$, whose wavefunction is plotted in figure 6 . 


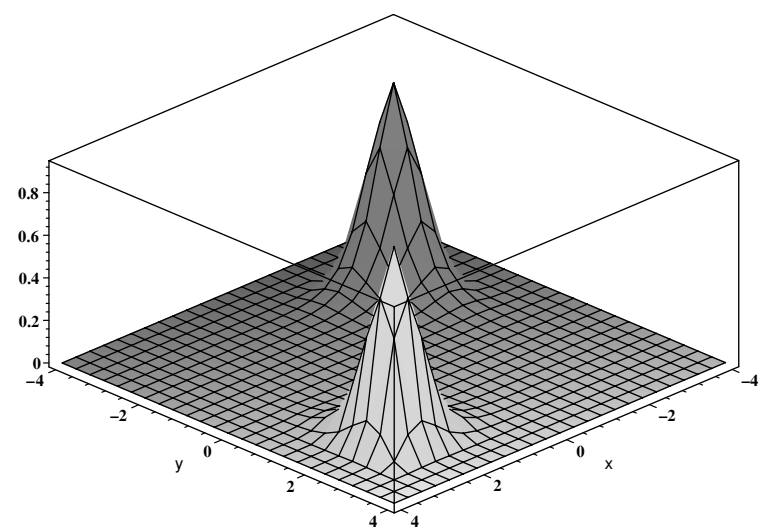

Figure 6. The wavefunction corresponding to the state $\left|\psi_{\text {ent }}\right\rangle^{\perp}$. The wavefunction and the spatial coordinates $x_{1,2}$ are depicted, respectively, in units of $\kappa$ and $1 / \kappa$ (see the appendix for details).

In this case, the actual time needed to reach an orthogonal state coincides with the bound given by (14),

$$
\begin{aligned}
\tau\left(\left|\psi_{\mathrm{ent}}\right\rangle\right) & =\tau_{\min }\left(\left|\psi_{\mathrm{ent}}\right\rangle\right) \\
& =\frac{\pi \hbar}{2\left(E_{1}-E_{0}\right)} .
\end{aligned}
$$

In other words, the entangled state $\left|\psi_{\text {ent }}\right\rangle$ saturates the bound (14).

It is instructive to compare some general aspects of the time evolution of states $\left|\psi_{R R}\right\rangle$ and $\left|\psi_{\text {ent }}\right\rangle$. In the case of the separable state $\left|\psi_{R R}\right\rangle$, it is possible to associate the individual pure state $\left|\psi_{R}\right\rangle$ with each particle, and these single-particle states evolve in such a way that the associated wavefunctions are localized alternately in each well. In other words, the expectation values $\left\langle x_{1,2}\right\rangle$ oscillate between the two wells. On the contrary, in the case of the entangled state $\left|\psi_{\text {ent }}\right\rangle$ each particle is described by the mixed state

$$
\rho_{1,2}=\frac{1}{2}\left(\left|\psi_{0}\right\rangle\left\langle\psi_{0}|+| \psi_{1}\right\rangle\left\langle\psi_{1}\right|\right)=\frac{1}{2}\left(\left|\psi_{L}\right\rangle\left\langle\psi_{L}|+| \psi_{R}\right\rangle\left\langle\psi_{R}\right|\right)
$$

These density matrices describing the single particle states do not evolve in time. The associated probability densities have two peaks, one in each well. The expectation values $\left\langle x_{1,2}\right\rangle$ are constant in time. In order to 'detect' the time evolution of the two-particle system one has to consider the behaviour of the expectation values of operators involving both particles. For instance, the expectation value

$$
\left\langle\left(x_{2}-x_{1}\right)^{2}\right\rangle
$$

exhibits a periodic time dependence. It adopts its maximum value for the wavefunction $\left|\psi_{\text {ent }}\right\rangle$ depicted in figure 5, and its minimum value for the orthogonal wavefunction $\left|\psi_{\text {ent }}\right\rangle^{\perp}$ depicted in figure 6.

\subsection{More general states}

The general state of our effective 2-qubit system is

$$
|\psi\rangle=a_{1}\left|\psi_{0}\right\rangle\left|\psi_{0}\right\rangle+a_{2}\left|\psi_{0}\right\rangle\left|\psi_{1}\right\rangle+a_{3}\left|\psi_{1}\right\rangle\left|\psi_{0}\right\rangle+a_{4}\left|\psi_{1}\right\rangle\left|\psi_{1}\right\rangle
$$

where the $a_{i}$ s are complex coefficients satisfying the normalization requirement,

$$
\left|a_{1}\right|^{2}+\left|a_{2}\right|^{2}+\left|a_{3}\right|^{2}+\left|a_{4}\right|^{2}=1 \text {. }
$$


The time $\tau$ needed for this state to evolve into an orthogonal one is given by

$$
\begin{aligned}
\left\langle\psi\left(t_{0}\right) \mid \psi\left(t_{0}+\tau\right)\right\rangle & =\mathrm{e}^{-2 \mathrm{i} E_{0} \tau / \hbar}\left[\left|a_{1}\right|^{2}+\left(\left|a_{2}\right|^{2}+\left|a_{3}\right|^{2}\right) \mathrm{e}^{-\mathrm{i}\left(E_{1}-E_{0}\right) \tau / \hbar}+\left|a_{4}\right|^{2} \mathrm{e}^{-2 \mathrm{i}\left(E_{1}-E_{0}\right) \tau / \hbar}\right] \\
& =0 .
\end{aligned}
$$

This equation for $\tau$ can be recast as

$$
P(x)=\left|a_{4}\right|^{2} x^{2}+\left(\left|a_{2}\right|^{2}+\left|a_{3}\right|^{2}\right) x+\left|a_{1}\right|^{2}=0
$$

with

$$
x=\mathrm{e}^{-\mathrm{i}\left(E_{1}-E_{0}\right) \tau / \hbar}
$$

The initial state (29) evolves to an orthogonal state if and only if the quadratic equation (32) admits at least one root with modulus equal to 1 . Let us consider the family of states (which we denote the ' $\beta$-family') leading to an equation (32) with two complex conjugate roots with modulus equal to 1 ,

$$
\mathrm{e}^{ \pm \mathrm{i} \beta}, \quad \text { with } \quad \beta=\left(E_{1}-E_{0}\right) \tau / \hbar \in[\pi / 2, \pi] .
$$

If that is the case, we can rewrite the polynomial $P(x)$ under the guise

$$
P(x)=\left|a_{4}\right|^{2}\left(x-\mathrm{e}^{\mathrm{i} \beta}\right)\left(x-\mathrm{e}^{-\mathrm{i} \beta}\right) .
$$

Comparing now expressions (32) and (35) for $P(x)$, and taking into account the normalization requirement (30), one obtains

$$
\left|a_{1}\right|^{2}=\left|a_{4}\right|^{2}=\frac{1}{2(1-\cos \beta)}, \quad\left|a_{2}\right|^{2}+\left|a_{3}\right|^{2}=-\frac{\cos \beta}{1-\cos \beta} .
$$

We thus see that the $\beta$-family of states evolving to an orthogonal state constitute a monoparametric family parameterized by the parameter $\beta$. Therefore, all the relevant quantities concerning these states can be written in terms of $\beta$. In particular,

$$
\tau_{\min }=\frac{\pi \hbar}{2 \Delta E}=\frac{\pi \hbar}{2\left(E_{1}-E_{0}\right)} \sqrt{1-\cos \beta},
$$

and the quotient between the actual time $\tau$ of evolution to an orthogonal state (which is related to $\beta$ through equation (34)) and the lower bound $\tau_{\min }$ is

$$
\frac{\tau}{\tau_{\min }}=\frac{2 \beta}{\pi \sqrt{1-\cos \beta}} .
$$

It is plain from this last equation that the lower bound on $\tau$ is saturated (that is, $\tau / \tau_{\min }=1$ ) only in the case $\beta=\pi / 2$, corresponding to the maximally entangled state $\left|\psi_{\text {ent }}\right\rangle$ given by equation (22).

On the other hand, let us consider a separable pure state

$$
|\phi\rangle=\left|\phi_{a}\right\rangle \otimes\left|\phi_{b}\right\rangle,
$$

which evolves to an orthogonal state. In order for this to happen, at least one of the states $\left|\phi_{a, b}\right\rangle$ has to evolve to an orthogonal state. Let us assume that

$$
\left|\phi_{a}\right\rangle=c_{0}\left|\psi_{0}\right\rangle+c_{1}\left|\psi_{1}\right\rangle
$$

evolves to an orthogonal state. In that case, we have

$$
\left|c_{0}\right|^{2}+\mathrm{e}^{-\frac{\mathrm{i} \tau\left(E_{1}-E_{0}\right)}{\hbar}}\left|c_{1}\right|^{2}=0 .
$$

Equation (41) clearly implies that

$$
\left|c_{0}\right|^{2}=\left|c_{1}\right|^{2}, \quad \mathrm{e}^{-\frac{\mathrm{i} \tau\left(E_{1}-E_{0}\right)}{\hbar}}=-1,
$$


and consequently, if the state belongs to the $\beta$-family, we have $\beta=\pi$. Therefore, it follows from equation (38) that separable states in the $\beta$-family have the highest possible value of $\tau / \tau_{\min }$,

$$
\left(\frac{\tau}{\tau_{\min }}\right)_{\text {separable }}=\sqrt{2} .
$$

The separable state given by equations (15)-(16), which we have previously considered in connection with the two-particle system in a double well, is an example of a state corresponding to the case $\beta=\pi$. This state illustrates an important feature of separable states: separable states that are energetically symmetric (that is, the energy and the energy's uncertainty are shared evenly between the subsystems) do not saturate the bound (14). In fact, all energetically symmetric separable states that evolve to an orthogonal state correspond to the case $\beta=\pi$. It must be emphasized that the two particles that we are considering do not interact with each other, and that the entanglement of the system (which is conserved in time) is given by its initial state.

\section{Conclusions}

We have used a system of two non-interacting quantum particles in a double-well potential to illustrate the connection between the speed of quantum evolution and quantum entanglement. The entanglement is preserved in time, and is given by the initial state of the system. The time required by separable (energetically symmetric) initial states to reach an orthogonal state does not saturate the bound (14). On the contrary, there exist (energetically symmetric) maximally entangled states (within an effective 2-qubit description) that do saturate the bound. That is, they evolve as fast as it is permitted by the value of their energy dispersion $\Delta E$. States of intermediate entanglement were also considered, and we proved that within the $\beta$-family all the states that saturate the bound have maximum entanglement (however, not all states with maximum entanglement do saturate the bound).

The connection between speed of evolution and entanglement in a system of two particles in a double-well potential offers interesting opportunities to illustrate the concept of entanglement in university courses on quantum mechanics. On the one hand, this illustration is based upon a set of well-known ingredients (i.e. quantum double-well potential, tunnelling time, etc) that are usually covered in courses on quantum mechanics. On the other hand, this example provides a clear instance of what we might call a 'positive' feature of quantum entanglement, as contrasted with the 'negative' way in which entanglement is usually defined. Entangled states are normally defined in terms of what they are not: an entangled pure state is a state that cannot be factorized. Most of the 'positive' aspects of entanglement involve its role as a resource to implement novel, non-classical types of computation and communication processes. Alas, a discussion of these processes in a quantum mechanics course would require the introduction of various new concepts in information theory and computer science. On the contrary, the role played by entanglement in 'speeding up' the evolution of two particles in a double well requires mostly ideas that are already part of standard courses in quantum mechanics.

\section{Acknowledgments}

This work has received partial support by FONDECYT, grants 1051075. The financial assistance of the National Research Foundation (NRF; South African Agency) towards this 
research is hereby acknowledged. Opinions expressed and conclusions arrived at are those of the authors and are not necessarily to be attributed to the NRF.

\section{Appendix}

The considerations discussed in the present work hold for general double-well potentials. However, the pictures depicted in this paper correspond to the particular, exactly soluble potential [32]

$$
V(x)=\frac{\hbar^{2} \kappa^{2}}{2 m}\left[\frac{1}{8} \xi^{2} \cosh 4 \kappa x-4 \xi \cosh 2 \kappa x-\frac{1}{8} \xi^{2}\right],
$$

with $\xi=0.1$. The potential function (A.1) is plotted in figure 1, using for $V(x)$ units of $\frac{\hbar^{2} \kappa^{2}}{2 m}$ and for $x$ units of $1 / \kappa$.

The eigenfunctions of the ground and first excited states of the potential (A.1) (here we are measuring the spatial coordinate $x$ in units of $1 / \kappa$ which, of course, is tantamount to adopt $\kappa=1)$ are given by [32]

$$
\begin{aligned}
& \psi_{0}(x)=\mathrm{e}^{-\frac{1}{4} \xi \cosh 2 x}\left[3 \xi \cosh x+\left(4-\xi+2 \sqrt{4-2 \xi+\xi^{2}}\right) \cosh 3 x\right] \\
& \psi_{1}(x)=\mathrm{e}^{-\frac{1}{4} \xi \cosh 2 x}\left[3 \xi \sinh x+\left(4+\xi+2 \sqrt{4+2 \xi+\xi^{2}}\right) \sinh 3 x\right]
\end{aligned}
$$

The corresponding energy eigenvalues are

$$
E_{0,1}=\frac{\hbar^{2} \kappa^{2}}{2 m} \epsilon_{0,1}
$$

with

$$
\begin{aligned}
& \epsilon_{0}=-\xi-5-2 \sqrt{4-2 \xi+\xi^{2}}, \\
& \epsilon_{1}=\xi-5-2 \sqrt{4+2 \xi+\xi^{2}} .
\end{aligned}
$$

\section{References}

[1] Walck S N and Hansell N C 2001 Characterization and visualization of the state and entanglement of two spins Eur. J. Phys. 22 343-50

[2] Tommasini P, Timmermans E and Toledo Piza A F R 1998 The hydrogen atom as an entangled electron-proton system Am. J. Phys. 66 881-6

[3] de la Torre A C 1994 Contextuality in quantum systems Am. J. Phys. 62 808-12

[4] Ekert A and Knight P L 1995 Entangled quantum systems and the Schmidt decomposition Am. J. Phys. 63 415-23

[5] de la Torre A C and Dotson A C 1996 An entangled opinion on the interpretation of quantum mechanics Am.J. Phys. 64174

[6] Aravind P K 1996 Geometry of the Schmidt decomposition and Hardy's theorem Am. J. Phys. 64 1143-50

[7] Roston G B, Casas M, Plastino A and Plastino A R 2005 Quantum entanglement, spin 1/2 and the Stern-Gerlach experiment Eur. J. Phys. 26 657-72

[8] Penrose R 2004 The Road to Reality (London: Jonathan Cape)

[9] Taylor J C 2002 Hidden Unity in Nature's Laws (Cambridge: Cambridge University Press)

[10] Dehlinger D and Mitchell M W 2002 Entangled photons, nonlocality, and Bell inequalities in the undergraduate laboratory Am. J. Phys. 70 903-10

[11] Dehlinger D and Mitchell M W 2002 Entangled photon apparatus for the undergraduate laboratory Am. $J$. Phys. 70 898-902

[12] Paty M 1999 Are quantum systems physical objects with physical properties? Eur. J. Phys. 20 373-88

[13] Nielsen N and Chuang I L 2000 Quantum Computation and Quantum Information (Cambridge: University Press) 
[14] Lo H-K, Popescu S and Spiller T (ed) 1998 Introduction to Quantum Computation and Information (River Edge: World Scientific)

[15] Bouwmeester D, Ekert A and Zeilinger A (ed) 1998 The Physics of Quantum Information (Berlin: Springer)

[16] Bennett C H, Brassard G, Crepeau C, Jozsa R, Peres A and Wootters W K 1993 Teleporting an unknown quantum state via dual classical and Einstein-Podolsky-Rossen channels Phys. Rev. Lett. 70 1895-9

[17] Bennett C H and Wiesner S J 1993 Communication via one- and two-particle operators on Einstein-PodolskyRossen states Phys. Rev. Lett. $692881-4$

[18] Ekert A and Jozsa R 1996 Quantum computation and Shor's factoring algorithm Rev. Mod. Phys. 68 733-53

[19] Giovannetti V, Lloyd S and Maccone L 2006 Quantum metrology Phys. Rev. Lett. 96010401

[20] Galindo A and Martin-Delgado M A 2002 Information and computation: classical and quantum aspects Rev. Mod. Phys. 74 347-423

[21] Gemmer J, Michel M and Mahler G 2004 Quantum Thermodynamics (Berlin: Springer)

[22] Giovannetti V, Lloyd S and Maccone L 2003 The role of entanglement in dynamical evolution Europhys. Lett. 62 615-21

[23] Giovannetti V, Lloyd S and Maccone L 2003 Quantum limits to dynamical evolution Phys. Rev. A 67052109

[24] Batle J, Casas M, Plastino A and Plastino A R 2005 Connection between entanglement and the speed of quantum evolution Phys. Rev. A 72032337

[25] Batle J, Casas M, Plastino A and Plastino A R 2005 Maximally entangled mixed states and conditional entropies Phys. Rev. A 71024301

[26] Rigo A, Plastino A R, Plastino A and Casas M 2000 On the inference of entangled states: a maximum-entropyminimum-norm approach Phys. Lett. A 2701

[27] Jacak L 2000 Semiconductor quantum dots-towards a new generation of semiconductor devices Eur. $J$. Phys. 21 487-97

[28] Isham C J 2001 Lectures on Quantum Theory: Mathematical and Structural Foundations (London: Imperial College Press)

[29] Peres A 1993 Quantum Theory: Concepts and Methods (Dordrecht: Kluwer)

[30] Sakurai J J 1985 Modern Quantum Mechanics (New York: Addison-Wesley)

[31] Feynman R P, Leighton R B and Sands M L 1969 The Feynman Lectures on Physics vol 3 (New York: Addison-Wesley)

[32] Razavy M 1980 An exactly soluble Schrödinger equation with a bistable potential Am. J. Phys. 48 285-8

[33] Vaidman L 1992 Minimum time for the evolution to an orthogonal quantum state Am. J. Phys. 60 182-3 